This document is the accepted manuscript version of the following article:

Bartkowiak, M., Zolliker, M., Ma, W., \& van den Brandt, B. (2019). A versatile, ultra-low background magnet for neutron scattering at SINQ. Journal of Neutron Research, 21(1-2), 59-64. https://doi.org/10.3233/JNR-190107

\title{
A versatile, ultra-low background magnet for neutron scattering at SINQ
}

\author{
Marek Bartkowiak ${ }^{\mathrm{a}, *}$, Markus Zolliker ${ }^{\mathrm{a}}$ Wenbin Ma ${ }^{\mathrm{b}}$ and Ben van den Brandt ${ }^{\mathrm{a}}$ \\ ${ }^{a}$ Laboratory for Scientific Developments and Novel Materials (LDM), Paul Scherrer Institut (PSI) Switzerland \\ E-mail: marek.bartkowiak@psi.ch \\ ${ }^{\mathrm{b}}$ Oxford Instruments NanoScience, Tubney Woods, Abingdon, Oxon OX13 5QX, UK
}

Abstract. The increasing efforts in material science to create new and purpose tailored materials and the deeper understanding of the complexity of condensed matter represent a major driving force for science constantly pushing the limits towards extreme experimental conditions and towards experiments with higher degree of complexity. The ability to develop and build new SE setups enables novel experiments with neutron scattering instruments. We present a modern, compact split-pair cryomagnet with a unique combination which maximises neutron beam access avoiding support structures in the beam to minimise the background. It provides a large 50 mm diameter sample space in a compact outer diameter for magnetic fields of up to 10 Tesla. The system does not require a lambda-stage and allows fast ramping.

Keywords: cryogenics, magnetic field, neutron absorber, borated aluminium, low temperature, cryomagnet

Neutron scattering techniques are an indispensable microscopic tool to study the structure of condensed matter, its interactions and elementary excitations. They are unmatched when studying magnetism. To gain insights in the working mechanisms of low dimensional magnetism, frustrated spin systems and quantum spin systems such as spin liquids and their magnetoelastic modes or spin chains as well as multifunctional materials such as multiferroics or topological magnets (skyrmions) a precise control of many external parameters such as temperature pressure or magnetic field is a prerequisite facilitated by the sample environment (SE).

The progress in our understanding of nature is ultimately tied to the advances in instrumentation and the ability to push external parameters beyond today's limits or to combine them in previously impossible ways. It is the latter where we see a huge potential for a wealth of experiments in a broad range of scientific research areas.

In many cases the life cycle of a sample follows a series of consecutive studies with several neutron scattering techniques, such as diffraction, spectroscopy/TOF and SANS under the same experimental conditions. It is therefore desirable but also economical to have equipment that can be used on different instruments.

For the magnet presented in this work the following combinations of external parameters have been considered:

(1) a high magnetic field, low temperature and high pressure

(2) a high magnetic field, low temperature combined with an electric field

(3) a high magnetic field for samples at ambient temperatures

Possibly the most important design parameter for the magnet is the available sample space in order to gain flexibility and allow for complex setups. By contrast, a small food print is also desirable to fit on as many instruments as possible. The beam access should be maximised and the material in the beam path reduced to a minimum.

Looking at the requirements it becomes clear why the combination of these conditions is extreme. Large magnetic fields are reached in small cold bore magnets, but do not permit large opening angles. This restrict the possibilities to implement sophisticated thermal or mechanical sample manipulation and limit the sample size to an extend that makes certain experiments impractical or impossible.

\footnotetext{
*Corresponding author. E-mail: marek.bartkowiak@psi.ch.
} 


\section{Scattering Geometry}

Since we wanted a magnet that can be operated at all scattering instruments at SINQ the outer dimensions of the magnet dewar are limited. At beam height the dewar has a diameter of $444 \mathrm{~mm}$ which is only $4 \mathrm{~mm}$ smaller than the space given by the radial collimator of the time of flight spectrometer FOCUS.

We settled with a split-pair magnet with a $10 \mathrm{~T}$ vertical field, a bore diameter of $\varnothing 70 \mathrm{~mm}$ and a split of $42 \mathrm{~mm}$. The two halves of the magnet are kept apart by three pillars, two $20^{\circ}$ and one $40^{\circ}$ leaving 3 large openings in the magnet former (see Fig. 1a,b). The surfaces of these openings were patterned with taped holes to attach shielding material. With a foreseen thickness of $1 \mathrm{~mm}$ shielding material two openings with $80^{\circ}$ and one with $110^{\circ}$ in the horizontal plane (see Fig. 1b) are obtained. The vertical opening is $\pm 10^{\circ}$ for a sample of $15 \mathrm{~mm}$ height and $20 \mathrm{~mm}$ diameter. The open geometry of the magnet with less than $3 \mathrm{~mm}$ of aluminium in the beam path enables measurements with extremely low background making it a viable choice for time of flight measurements or enabling measurements on small samples. For interesting exotic spin states, for example, the combination of a high field and a low background is crucial. a)
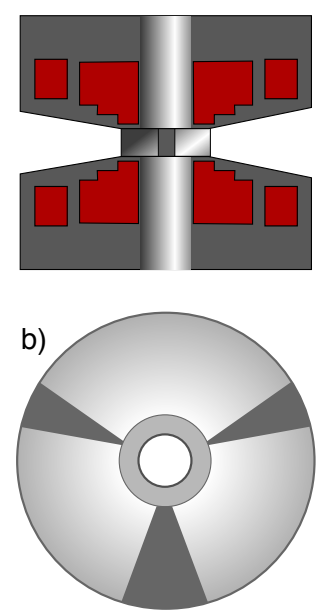

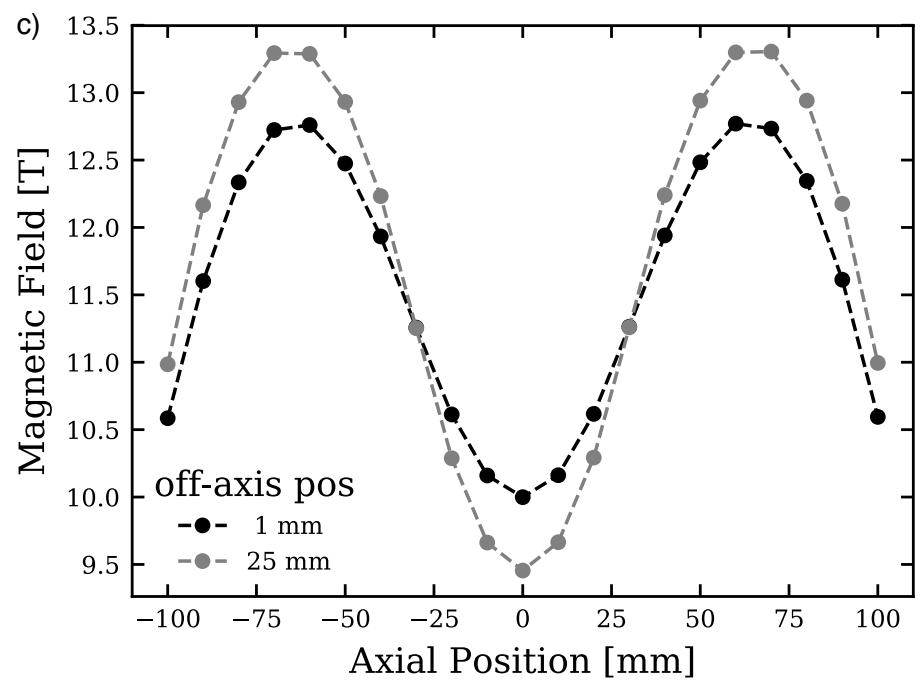

. 1. a) The vertical cross section of magnet with a vertical split of $42 \mathrm{~mm}$ and an opening angle of $\pm 10^{\circ}$. b) View of the center plane the magnet. The tree gray triangles indicated the support structure of the magnet split providing beam access for $2 \times 80^{\circ}$ and $110^{\circ}$. c) Axial magnetic field on center line and $25 \mathrm{~mm}$ off-axis for a nominal field of $10 \mathrm{~T}$ at the sample.

The large vertical opening of the magnet (see Fig. 1a) make it even suitable for small angle scattering experiments. The limited access within the scattering plane can be compensated utilising independent rotation of the magnet and the sample around the magnetic field axis. For single detector systems there is no drawback and all scattering configurations can be reached. For area detectors the observable q-range is limited by the 110 deg given by the largest window.

\section{Magnet Specifications}

The magnet has two halves, each half is optimally configured with concentric Nb3Sn sections surrounded by an outer NbTi section. It can be operated in symmetric and asymmetric mode reaching a maximum field of $10 \mathrm{~T}$ and $9.25 \mathrm{~T}$ respectively. The magnet can reach field maximum within $20 \mathrm{~min}$ and without the use of a lambda stage which results in easy and fast operation. A trade-off for the wide vertical opening angle and the large split is the relatively low homogeneity. Over a cylindrical sample volume of $20 \mathrm{~mm}$ diameter and $15 \mathrm{~mm}$ height the field 
homogeneity is only $1.75 \%$ and $2.5 \%$ for the symmetric and asymmetric mode. Figure $1 \mathrm{c}$ shows the field strength along the axial line and with a radial offset of $25 \mathrm{~mm}$.

The asymmetric mode is achieved by disabling one of the sections in the lower half of the magnet. This moves the zero flux density node $30 \mathrm{~mm}$ below the beam and results in a maximum depolarisation factor $\frac{d \theta}{d L} B^{-1}$ of $435 \mathrm{rad} / \mathrm{Tm}$ for a radial path $7.5 \mathrm{~mm}$ below the scattering plane and a central field of $2 \mathrm{~T}$. This is well below the required inequality $\omega_{B} / \omega_{L}<0.1$ [1], [2] for any wavelength used at SINQ, where $\omega_{B}$ is the rate of the angular rotation of the magnetic field and $\omega_{L}$ denotes the Lamor frequency of the neutron.

\section{Neutron Shielding}

The open geometry of the magnet forms the basis of the strategy to reduce the background related to the sample environment. To take full advantage of this concept the magnet former needs to be sufficiently shielded against the diverging incoming beam as well as the scattered outgoing beam. This is achieved by covering the surfaces with a neutron absorbing layer. The materials most commonly used are gadolinium, cadmium, lithium or boron.

The absorption length for gadolinium of only about $7 \mu \mathrm{m}$ for thermal neutrons allows very effective screening with only a few $10 \mu \mathrm{m}$ coverage. This is achieved by painting with Gd-containing paint or sputtering. The radiation of prompt gammas during the absorption, however, is an unavoidable side effect which is acceptable for the low intensity secondary beam but not for shielding the primary beam.

Cadmium is also very often used, in particular as a material to build temporary shielding near the sample position. It is very soft and easy to shape, but care needs to be taken to make sure that it does not deform over time. Using a soft metal like cadmium in close proximity to superconducting magnet bears the risk of deformation under quench forces.

Boron containing materials like boron nitride have also been used for shielding purposes at cryogenic temperatures. Here, only the isotope ${ }^{10} \mathrm{~B}$ has a high absorption cross-section with a natural abundance of $20 \%$. The absorption length of boron nitride for thermal neutrons is about $1 / 4 \mathrm{~mm}$ which does allow the design of thin shielding layers. However, it is extremely hard and brittle. Hence, it is difficult to machine and care has to be taken to reduce thermally induced stress when using it in a cryogenic environment.

For the magnet presented in this work we used ${ }^{10} \mathrm{~B}$ enriched borated aluminium [3] (AB45) with $4.5 \%$ weight percent of boron and $>95 \%$ enrichment. This alloy has neutron absorption properties which are comparable to Boral ${ }^{\circledR}$ with a boron content of $25 \%$. Sheets of this material with a thickness of $1 \mathrm{~mm}$ were used, which could easily be cut and bend into shape. The magnet former with the installed shielding is shown in Fig. $2 b$. The shielding is held in place by aluminium screws that screw into tapped holes in the magnet former which were added during production.

The surfaces of the magnet spacer columns which are facing the sample can be exposed to the direct neutron beam. It can be assumed that the neutron beam always strikes parallel to the surface normal $\left(\theta=90^{\circ}\right)$ and additional shielding material is required. For the thinner columns, we bend the radially mounted sheets slightly inwards to increase the apparent absorber thickness. For the wide column, in total 3 sheets of borated aluminum where folded to form a thick absorber block (see Fig. 2c)

The absorption of neutrons is described by the Beer-Lambert law [4] $I=I_{0} \exp (-\Sigma d)$, where $I_{0}, I, \Sigma$ and $d$ are the initial intensity, the intensity after transmission, the linear absorption coefficient and the absorber thickness respectively. The absorption length is therefore defined as $l_{a b s}=\Sigma^{-1}$.

Using the wave length dependence of the neutron absorption cross section [5] the absorption length for AB45 can be calculated. It is shown by the black curve in Fig. 2a, without considering the absorption of aluminium and neglecting the density differences of the parent compounds. Here, we included the curve (gray) of boron nitride for reference. The dashed line in Fig. 2a shows the wavelength dependence of the incident angle $\theta$ where the apparent thickness of the shielding material reaches $7 \times l_{a b s}$. This corresponds to a reduction of the transmission by a factor 1000 . 


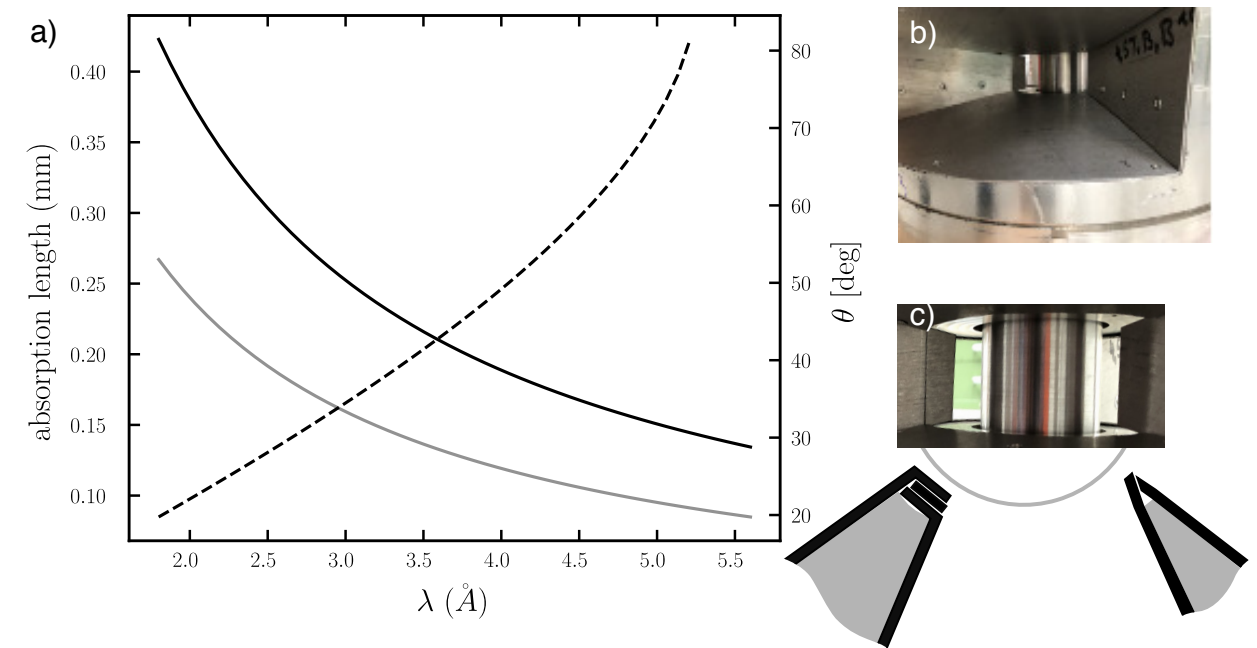

Fig. 2. a) The calculated wavelength dependence of the scattering length for Bor-Aluminium with $4.5 \%{ }^{10} \mathrm{~B}$ (solid black line) and Boron Nitride (solid gray). The dashed line shows the take-off angle for neutrons penetrating the shielding with an apparent thickness of $7 \times$ scattering length. b) A section of the magnet former with neutron shielding installed. c) The sample facing surfaces of the magnet former are strengthened to suppress the impact of the direct beam. The schematic top view indicates the geometrical arrangement.

\section{Shielding Performance}

The magnet was measured on the time of flight spectrometer FOCUS at SINQ using the same setup, with and without shielding in place. The selected neutron wavelength was $5 \AA$. In figure $3 \mathrm{a}-\mathrm{b}$, the recorded detector signal is shown as a function of scattering angle and flight time. The data on the left hand side (Fig. 3a ) were taken before the shielding was installed. In addition to the intensity of the elastic line at $4.5 \mathrm{msec}$, spurious signals can be found in the vicinity of the line. In addition, some non-negligible intensity is observed at angles $\theta>110^{\circ}$. At these angles the detector is shadowed by one of the magnet columns and no signal should be observed. After the installation of the shielding material (Fig. 3b) the spectrum looks much cleaner and all the spurious features are suppressed. Fig. $3 \mathrm{c}$ shows the magnet installed on the instrument.

\section{Cryostat and Upgrade Options}

The magnet is equipped with a variable temperature insert (VTI) with an inner diameter of $50 \mathrm{~mm}$ over the entire length of the sample space. The VTI is operated with static exchange gas and the distance between the heat-exchanger and the magnet center is $220 \mathrm{~mm}$ with a clear depth of $120 \mathrm{~mm}$ below the beam position. The temperature at the sample can be varied between $1.5 \mathrm{~K}$ to $300 \mathrm{~K}$. The available space makes it also possible to place sample holders with additional capabilities.

The use of our existing $3 \mathrm{He}-4 \mathrm{He}$ dilution refrigerators (KelvinoxVT) expands the available temperature range down to $<50 \mathrm{mK}$ reducing the available sample space diameter to $40 \mathrm{~mm}$. The cryostat is fully compatible with our electric field stick [6] which combines the temperature and magnetic field capabilities with the possibility to apply up to $5 \mathrm{kV}$ across the sample. The magnet can be combined with non-magnetic piston cylinder pressure cells, which were developed by R. Sadykov, Troitsk, Russia, and reach pressures as high as $>10 \mathrm{kbar}$. 

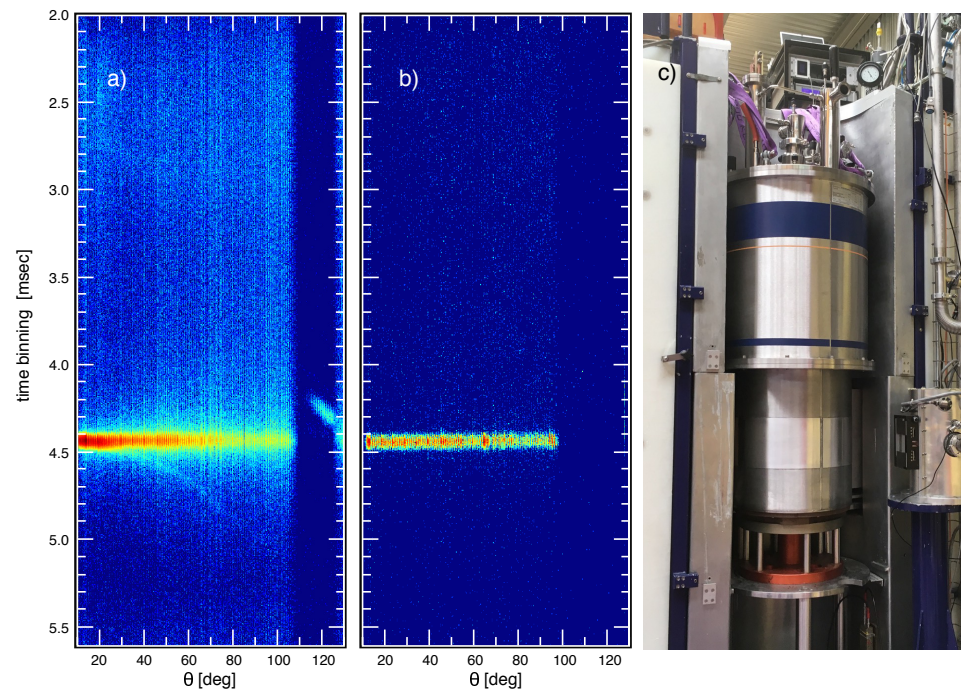

Fig. 3. Time of flight spectrum measured on FOCUS without a) and with b) neutron shielding in place. c) The magnet installed on FOCUS.

\section{Summary and Outlook}

We presented a modern, compact split-pair cryomagnet with a unique combination of available sample space, reachable scattering angles and magnetic fields. The combination of large sample space and low background will path the way for spectroscopic studies of magnetic phenomena under pressure. The low background of the magnet is advantageous for demanding spectroscopy experiments. In addition it enhances the resolution for determining magnetic form factors of magnetization in diffraction experiments. The opening angles are chosen with foreseen SINQ instrument upgrades in mind to exploit their full potential right from the beginning of their operation. Moreover, they will enable research on magneto-responsive bio-materials whose properties can be tuned by an external magnetic field, which serves as a first step towards the development of smart biomaterials.

\section{Acknowledgements}

We thank Paul Schurter, Stephan Fischer and Silvan Stamm for their work and support during the installation and integration of the system. It is highly appreciated what they did during a fully running cycle of the facility. A special thank goes to J. P. Embs, who kindly performed the background measurements on FOCUS. This work has been supported by the SNSF under the R'EQUIP grant No. 157809.

\section{References}

[1] S. R., Polarized Neutrons, 2011. http://www.oxfordneutronschool.org/2011/lectures/osns_stewart_polarised_2011.pdf.

[2] M. Maldonado-Velázquez, L. Barrón-Palos, C. Crawford and W.M. Snow, Nucl. Instrum. Methods Phys. Res. A 854 (2017), 127-133. doi:https://doi.org/10.1016/j.nima.2017.02.066.

[3] ceradyne, borated aluminium, 2702 Boulevard Talbot, Chicoutimi, Quebec G7H 5B1, CANADA, 2019. https://multimedia.3m.com/mws/ media/999486O/3m-stable-isotopes.pdf.

[4] A.J. Dianoux and G.H. Lander, Neutron Data Booklet, Old City Publishing, Incorporated, 2003. ISBN 9780970414373.

[5] M.F. L'Annunziata, in: Handbook of Radioactivity Analysis (Third Edition), Third edition edn, M.F. L'Annunziata, ed., Academic Press, Amsterdam, 2012, pp. 1-162. doi:https://doi.org/10.1016/B978-0-12-384873-4.00001-3.

[6] M. Bartkowiak, J.S. White, H.M. Rønnow and K. Prša, Rev. Sci. Instrum. 85(2) (2014), 026112. doi:10.1063/1.4865406. 